\title{
Proximal pulmonary emboli modify right ventricular ejection pattern
}

\author{
A. Torbicki*, M. Kurzyna*, M. Ciurzynski**, P. Pruszczyk**, R. Pacho***, \\ A. Kuch-Wocial**, M. Szulc**
}

Proximal pulmonary emboli modify right ventricular ejection pattern. A. Torbicki, M. Kurzyna, M. Ciurzynski, P. Pruszczyk, R. Pacho, A. Kuch-Wocial, M. Szulc. (C)ERS Journals Ltd 1999.

ABSTRACT: Analysis of the systolic flow velocity curve (SFVC) in the right ventricular outflow tract is considered as an alternative to the tricuspid valve pressure gradient (TVPG) method for echo-Doppler assessment of pulmonary arterial pressure (Ppa). The present study checked whether or not SFVC is affected by the cause of pulmonary hypertension.

Doppler recordings of 86 patients (39 female, aged 55.5 \pm 15.2 yrs) with acute (APPE) or chronic (CP-PE) proximal pulmonary embolism, chronic obstructive pulmonary disase (COPD) or primary pulmonary hypertension (PPH) were retrospectively analysed by two observers unaware of the purpose of the study.

Despite having the lowest TVPG $(48 \pm 13 \mathrm{mmHg})$, patients with AP-PE had the shortest acceleration time ( $t$ acc; $56 \pm 15 \mathrm{~ms})$ and time to midsystolic deceleration $(t \mathrm{msd}$; $105 \pm 16 \mathrm{~ms}$ ). $t$ acc $<60 \mathrm{~ms}$ in patients with TVPG $<60 \mathrm{mmHg}$ had $98 \%$ specificity and $48 \%$ sensitivity for AP-PE. In PPH, SFVC was less abnormal (tace $64 \pm 14 \mathrm{~ms}$, $t \mathrm{msd}$ $125 \pm 25 \mathrm{~ms}$, both $\mathrm{p}<0.03)$ despite having a TVPG twice as high $(92 \pm 12 \mathrm{mmHg}, \mathrm{p}<$ $0.001)$. In contrast to tacc, TVPG showed strong correlation with direct $P$ pa measurements whenever performed $(r=-0.43, p=0.02$, versus $r=0.80, p<0.001 ; n=30)$. There was no correlation between tacc and TVPG in a pooled study group and SFVC seemed strongly affected by the presence of both AP-PE and CP-PE.

While potentially useful for evaluation of the true right ventricular afterload during pulsatile flow conditions, the systolic flow velocity curve does not provide a reliable estimate of pulmonary arterial pressure.

Eur Respir J 1999; 13: 616-621.

The method of choice for noninvasive estimation of pulmonary arterial pressure $(P \mathrm{pa})$ is based on continuous wave Doppler measurement of the peak velocity of the regurgitant jet across the tricuspid valve (tricuspid valve pressure gradient; TVPG). This method, based on the simplified Bernoulli equation and a straightforward pathophysiological concept, proved highly reliable in a wide spectrum of cardiovascular disease [1-4].

However, the pulsed wave Doppler-derived pattern of the systolic flow velocity curve (SFVC) in the right ventricular (RV) outflow tract is also believed to reflect the level of $P$ pa [5-9]. Using SFVC is appealing because, in contrast to TVPG, it can be recorded in almost every patient, including those with lung hyperinflation [10]. Coexistence of short acceleration time (tacc) and midsystolic deceleration ( $t \mathrm{msd}$; which has a "notched" pattern) is considered diagnostic of severe pulmonary hypertension [5]. However, such patterns have also been observed in the setting of acute pulmonary embolism [11] and similar changes have been induced experimentally by proximal constriction of the proximal pulmonary arteries in dogs [12]. In both these situations, marked SFVC changes were found in the presence of acute but relatively mild elevation of $P$ pa limited by the performance of nonhypertrophied RV.
*Dept of Internal Medicine, National Tuberculosis and Lung Disease Research Institute. Warsaw, Poland. **Dept of Internal Medicine and Hypertension, ***Division of Radiology, University School of Medicine, Warsaw, Poland.

Correspondence: A. Torbicki Dept of Internal Medicine

National Tuberculosis and Lung Disease Research Institute

ul. Plocka 26

01-138 Warsaw

Poland

Fax: 48226286737

Keywords: Doppler echocardiography pulmonary circulation pulmonary embolism pulmonary hypertension right ventricular function

Received: March 171998 Accepted after revision October 191998
In this study echo/Doppler findings were compared in patients with four clinical situations, all leading to precapillary pulmonary hypertension, but differing either with regard to the site of vascular obstruction, severity of hypertension or mode of onset. It was expected that such a comparison may help to understand the determinants of SFVC and, hopefully, assist in the differential diagnosis of pulmonary hypertension in the future.

\section{Patients and methods}

The study was performed in a teaching hospital by a team dedicated to the management of patients with unexplained pulmonary hypertension.

A retrospective analysis of technically adequate echocardiographic tracings was performed in order to identify patients with RV systolic overload, defined as a tricuspid jet velocity of $>2.8 \mathrm{~m} \cdot \mathrm{s}^{-1}$ measured with colour-guided continuous wave Doppler and averaged over five consecutive heart cycles. This velocity, according to the simplified Bernoulli equation, corresponds to an RV systolic pressure of $>30 \mathrm{mmHg}$.

Patients were excluded from further study if echocardiographic evaluation suggested left heart disease, 
pulmonary valvular stenosis or intracardiac shunt as a potential cause of RV pressure overload. Patients were included in the study if during their clinical evaluation enough evidence was collected to allow a reliable diagnosis of acute proximal pulmonary embolism (AP-PE), chronic proximal pulmonary embolism (CP-PE), chronic parenchymal lung disease (CPLD) or primary pulmonary hypertension (PPH).

Proximal pulmonary embolism was diagnosed if thromboemboli could be unequivocally visualized in the main or lobar pulmonary arteries with either contrast enhanced spiral computer tomography (s-CT) or transoesophageal echocardiography (TOE). The detailed methods applied and criteria used for identification of thromboemboli have been previously described [13]. In all cases, diagnosis of pulmonary embolism was additionally made by either high probability lung scan or pulmonary angiography, as appropriate. Acute pulmonary embolism was diagnosed only if clinical and echocardiographic signs of RV overload were no longer found after 3 months of antithrombotic treatment. Chronic pulmonary embolism was diagnosed only when the pulmonary arterial thrombi and signs of RV pressure overload persisted for at least 3 months of antithrombotic treatment following initial evaluation. CPLD was diagnosed according to clinical criteria and assessment of lung mechanics, when appropriate. Unexplained pulmonary hypertension or PPH were diagnosed when extensive diagnostic evaluation including the methods mentioned above failed to disclose factors responsible for elevated RV afterload. Thirty patients among those with CP-PE, CPLD and $\mathrm{PPH}$ had invasive right heart pressure measurements with fluid-filled catheters within a month of index echocardiographic examination. In patients with confirmed AP$\mathrm{PE}$, invasive studies were withheld in order to decrease bleeding complications of thrombolytic treatment, if required.

The echo/Doppler tracings of patients who were considered suitable for further assessment were blindly reevaluated by two specially instructed sixth year medical students (M. Kurzyna and M. Ciurzynski) who were unaware at the time of the purpose of the study and clinical diagnosis of the patients. This evaluation consisted of a detailed analysis of pulsed wave Doppler flow velocity tracings recorded with the lowest possible wall filter setting at a sweep speed of $100 \mathrm{~cm} \cdot \mathrm{s}^{-1}$ during spontaneous respiration in the RV outflow tract, just below the level of the pulmonary valve. The detailed method of such registrations used in this laboratory has been previously described $[10,14-16]$. Importantly, the sample volume of the pulsed wave Doppler is placed under two-dimensional (2-D) and colour-coded Doppler guidance in the main stream of the RV ejection just below the level of the pulmonary valve. Also, the standardization of the sample volume position with regard to the pulmonary valve is enhanced by searching for a typical SFVC pattern, free from the signal of opening but displaying a clear signal of the closing pulmonary valve leaflets.

The following parameters were measured (fig. 1): tacc defined as the time interval from the onset of forward flow into the pulmonary artery to the peak velocity of this flow; tmsd defined as the time interval from the onset of forward flow to the moment of maximum midsystolic deceleration (when present); right ventricular ejection time (RVET), defined as the time interval from the onset of forward flow to the closure of the pulmonary arterial valve; right ventricular ejection integral (RVEI), defined as the area enclosed within the flow velocity curve recorded in the RV outflow tract (RVOT) during the time of RV ejection; integral-to-notch (Nint), defined as the area enclosed within the flow velocity curve of the RV ejection from its onset to the moment of maximum midsystolic deceleration, whenever present.

Reproducibility of the Doppler-derived RV time intervals and integrals in the laboratory were previously checked in a group of 30 patients with suspected chronic cor pulmonale [17]. The mean \pm SD difference between measurements taken in the same patient on two consecutive days were $7.6 \pm 6.3 \mathrm{~ms}$ for $\mathrm{tacc}$, and $0.91 \pm 0.74 \mathrm{~ms}$ for RVEI when taken by a single observer and $9.1 \pm 6.0 \mathrm{~ms}$ and $0.54 \pm 0.55 \mathrm{~ms}$, respectively, when taken by two independent observers. In order to compensate for eventual differences in cardiac frequency between the studied groups, two indexes were also calculated: tacc/RVET(\%) and Nint/ RVEI (\%).

All measurements were averaged over at least five consecutive heart cycles. If midsystolic decelerations were not present in every heart cycle the number of cycles with midsystolic decelerations were counted over 10 consecutive heart cycles. Measurements requiring the presence of midsystolic decelerations ( $t \mathrm{msd}$, Nint) were then averaged
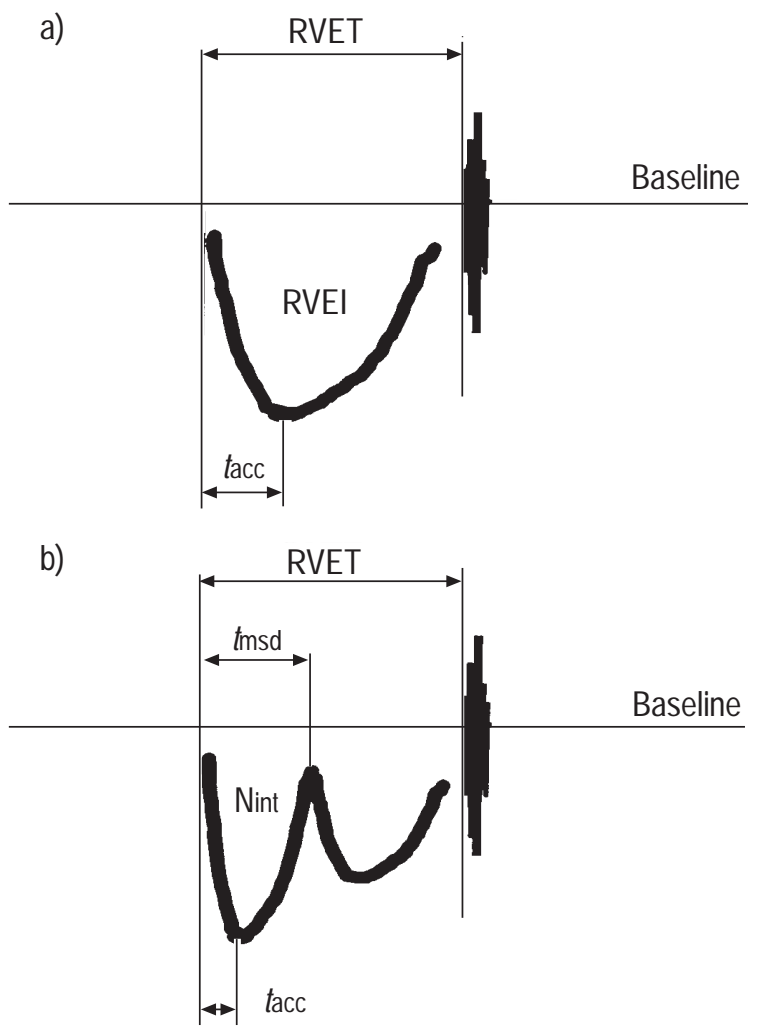

Fig. 1. - Schematic presentation of measurements performed on the systolic flow-velocity curve (SFVC). Two types of tracings are presented: a) without and b) with midsystolic deceleration ( $t \mathrm{msd})$. Note that the leading edge of the earliest recorded flow signal was considered as the onset of forward flow while the leading edge of the earliest signal from closing pulmonary valve was considered as termination of forward flow. tacc: acceleration time; RVET: right ventricular ejection time; RVEI: right ventricular ejection integral; Nint: integral in the part of ejection from onset to "notch". 
Table 1. - Selected clinical characteristics of the studied patients

\begin{tabular}{lccccc}
\hline & AP-PE & CP-PE & CPLD & PPH & p-value* \\
\hline n & 25 & 26 & 23 & 12 & \\
Age yrs & $68 \pm 8^{\ddagger, \#, \S}$ & $52 \pm 14^{\S}$ & $55 \pm 13^{\S}$ & $38 \pm 12$ & 0.00001 \\
fC beats.min & $102 \pm 19$ & $89 \pm 16$ & $99 \pm 17$ & $97 \pm 30$ & NS \\
IVC mm & $22 \pm 6.6$ & $24 \pm 6.1$ & $21 \pm 4.5$ & $22 \pm 3.7$ & NS \\
RVedd & $33 \pm 6$ & $37 \pm 6$ & $33 \pm 10$ & $37 \pm 6$ & NS \\
$P$ pa mmHg & & $87 \pm 18$ & $48 \pm 17$ & $102 \pm 10$ & \\
TVPG mmHg $^{\dagger}$ & & $82 \pm 20$ & $52 \pm 13$ & $94 \pm 9$ & \\
\hline
\end{tabular}

$\dagger$ : Data calculated for 30 patients with direct pulmonary arterial pressure measurements. AP-PE: acute proximal pulmonary embolism; CP-PE: chronic proximal pulmonary embolism; CPLD: chronic parenchymal lung disease; PPH: primary pulmonary hypertension; $f \mathrm{C}$ : cardiac frequency; IVC: inferior vena cava (diameter); RVedd: right ventricular end-diastolic dimension; $P$ pa: pulmonary artery systolic pressure; TVPG: tricuspid valve pressure gradient. *: As assessed by ANOVA. In addition differences between the subgroups are indicated, whenever present; ${ }^{\star}: \mathrm{p}<0.01$ versus CP-PE; ${ }^{\#}: \mathrm{p}<0.01$ versus $\mathrm{CPLD} ;{ }^{\S}$ : $\mathrm{p}<0.01$ versus $\mathrm{PPH}$.

over those cycles, in which midsystolic decelerations was actually present.

In order to assess RV preload conditions, twodimensional guided M-mode echocardiographic measurements were performed of the RV end-diastolic dimension (RVedd), using a parasternal view; and inferior vena cava (IVC) at expiration from the subcostal view. The significance of the differences between the groups were compared with the one way analysis of variance (ANOVA) and Student's t-test and correlations were assessed by Pearson correlation coefficient.

\section{Results}

Altogether 86 patients were analysed, 39 females and 47 males, aged $55.5 \pm 15.2$ yrs. When divided according to the clinical diagnosis, the patients were found to differ with respect to age, which was lowest in $\mathrm{PPH}$ patients and highest in patients with AP-PE patients. Interestingly, cardiac frequency, dimensions of the RV and IVC were similar in all the subgroups (tables 1 and 2). In 30 patients who had direct pressure measurements, systolic $P$ pa correlated strongly with TVPG $(\mathrm{r}=0.80, \mathrm{p}<0.001)$, but poorly with $\operatorname{tacc}(\mathrm{r}=-0.43, \mathrm{p}=0.02)$. There was no correlation between mean $P$ pa and tacc $(\mathrm{r}=-0.34, \mathrm{p}=0.07)$.
Doppler-derived parameters differed markedly between the subgroups. Tricuspid jet velocity (TVPG) was highest in PPH patients, almost twice as high as in patients with AP-PE and this difference was highly significant $(88 \pm 12$ versus $48 \pm 13 \mathrm{mmHg}$, respectively, $\mathrm{p}<0.001$ ). Paradoxically the most disturbed SFVC curve was found in patients with AP-PE with a tacc of $56 \pm 15 \mathrm{~ms}$, significantly shorter than in the PPH group (64 $\pm 14 \mathrm{~ms}, \mathrm{p}=0.025)$. Also, tmsd was shortest in patients with AP-PE and there was a strong correlation between $t \mathrm{msd}$ and tacc in the pooled studied group $(\mathrm{r}=0.77, \mathrm{p}<0.0001)$. Midsystolic deceleration ("notch") was found in $100 \%$ and $96 \%$ of the patients with CP-PE and AP-PE, respectively, and in $56 \%$ and $83 \%$ of patients with CPLD and PPH, respectively. The tace/RVET (\%) was low and identical in both subgroups of patients with proximal pulmonary embolism, and higher, but very similar, in the two remaining subgroups. Surprisingly, there was no correlation between tacc and TVPG in the pooled study group. Instead, the measurements representing individual patients from each of the four studied subgroups clustered in different areas of the scatterplot (fig. 2). Short tacc $(<60$ $\mathrm{ms}$ ) occurring in patients without marked TVPG elevation $(<60 \mathrm{mmHg})$ seemed to be especially characteristic for AP-PE (specificity 98\%, sensitivity 48\%) (fig. 3).

\section{Discussion}

In the present study, TVPG and SFVC (universally considered as an alternative to Doppler methods of noninvasive evaluation of $P$ pa) were assessed in four subgroups of patients with different causes of precapillary pulmonary hypertension. In the pooled studied group there was an obvious dissociation between TVPG measurements and the direction of changes observed in the parameters derived from SFVC. In general, SFVC seemed to be strongly affected by the presence of proximal emboli in the pulmonary arterial bed. In fact, the correlation between tacc and either systolic or mean $P$ pa were weak, contrasting with the strong correlation confirmed between TVPG and directly measured systolic $P$ pa.

These findings are compatible with the concept of a pressure wave reflected from the peripheral pulmonary arteries [18]. In normal conditions, a reflected pressure wave returns late and hits the area of the pulmonary valve after the RV has completed its ejection [19]. This seems to be one of the mechanisms of optimal RV-pulmonary artery coupling. By increasing both the velocity and amplitude of the reflected wave, pulmonary hypertension causes its

Table 2. - Results of echo/Doppler assessment of the studied patients

\begin{tabular}{lccccc}
\hline & AP-PE & CP-PE & CPLD & PPH & p-value* \\
\hline TVPG mmHg & $48 \pm 13^{\S, \$}$ & $79 \pm 19^{\#, \$}$ & $55 \pm 13^{\S, 末}$ & $92 \pm 12$ & 0.00001 \\
tacc ms & $56 \pm 15$ & $59 \pm 17^{+}$ & $69 \pm 14$ & $64 \pm 14$ & 0.022 \\
$t$ acc/RVET \% & $22 \pm 7^{\#, \$}$ & $22 \pm 5^{\#, \$}$ & $29 \pm 8$ & $28 \pm 7$ & 0.0003 \\
$t$ msd ms & $105 \pm 16^{+, \S}$ & $112 \pm 21$ & $122 \pm 23$ & $125 \pm 25$ & 0.025 \\
$t$ msd/RVET \% & $41 \pm 8^{\#, \S}$ & $41 \pm 6^{\#, \S}$ & $50 \pm 7$ & $50 \pm 5$ & 0.0001 \\
Nint cm & $4.6 \pm 1.5^{\S}$ & $4.9 \pm 2.1^{\$}$ & $6.4 \pm 3.2$ & $6.7 \pm 2.4$ & 0.027 \\
RVEI cm & $9.8 \pm 4.2$ & $9.2 \pm 3.5$ & $11.7 \pm 4.9$ & $10.1 \pm 3.7$ & NS \\
Nint/RVEI \% & $52 \pm 8^{\S}$ & $54 \pm 6^{\$}$ & $56 \pm 7$ & $62 \pm 5$ & NS \\
\hline
\end{tabular}

*: As assessed by ANOVA. In addition differences between the subgroups are indicated, whenever present; ${ }^{\dagger}: \mathrm{p}<0.01$ versus $\mathrm{CP}-\mathrm{PE} ;{ }^{*}$ : $\mathrm{p}<0.01$ versus CPLD; ${ }^{+}$: $\mathrm{p}<0.05$ versus CPLD $^{\S}{ }^{\S}$ : $\mathrm{p}<0.01$ versus $\mathrm{PPH} ;{ }^{\$}$ : $\mathrm{p}<0.05$ versus PPH. tacc: acceleration time; tmsd: midsystolic deceleration time; RVET: right ventricular ejection time; Nint: integral to "notch"; RVEI: right ventricular ejection integral. For remainder of definitions see legend to table 1 . 


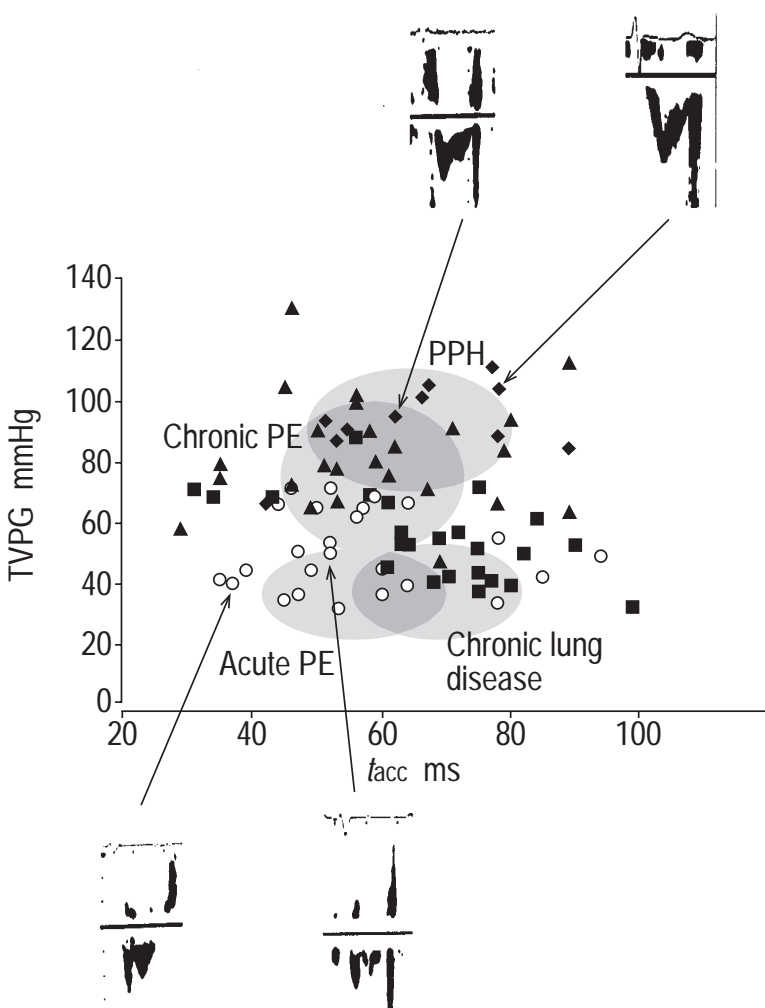

Fig. 2. - Lack of correlation between tricuspid valve pressure gradient (TVPG) and acceleration time (tacc). Note the clustering of observations regarding patients with the same causes of primary pulmonary hypertension $(\mathrm{PPH} ; \bullet)$ (the centre of the ellipsoid "clouds" indicate the mean and the axes indicate SD for the TVPG and tacc in individual subgroups of patients, respectively). Despite having the lowest TVPG, patients with acute proximal pulmonary embolism (AP-PE; O) often display a severely disturbed systolic flow-velocity curve (SFVC) (bottom tracings) even when compared to patients with PPH (top tracings), despite the highest TVPG found in the latter subgroup. $\mathbf{\Delta}$ : chronic proximal pulmonary embolism; $\mathbf{a}$ : chronic parenchymal lung diseases.

earlier return manifesting itself as midsystolic deceleration, found on Doppler-derived SFVC [12, 18-20]. While TuRKEVITCH et al. [21] found a correlation between the time-to-notch and the severity of pulmonary hypertension, it might also be affected by the site of vascular changes. FURUNO et al. [12] compared pulmonary pressure and flow responses in dogs submitted either to distal pulmonary embolization or proximal pulmonary constriction. Despite similar levels of acutely induced pulmonary hypertension, the SFVC in the latter group were characterized by an apparently shorter tacc and the presence of early systolic deceleration. The present clinical observations confirm that the site of obstruction affects SFVC pattern in patients with precapillary pulmonary hypertension. It is felt that in the presence of proximal thromboemboli, reflected pressure waves return very early resulting in an early "notch" which cuts off the peak of the flow velocity curve and therefore determines the duration of tacc. In the present study, this would be suggested by a close correlation between $t \mathrm{msd}$ and the tacc. The most marked dissociation between TVPG and tacc should then be expected when a proximally located obstruction site coincides with a relatively low $P$ pa. Among the four studied subgroups, AP-PE best fits this description.

Could the "TVPG $<60$, tacc $<60$ " sign be clinically helpful in the diagnosis of AP-PE? Sample volume position,
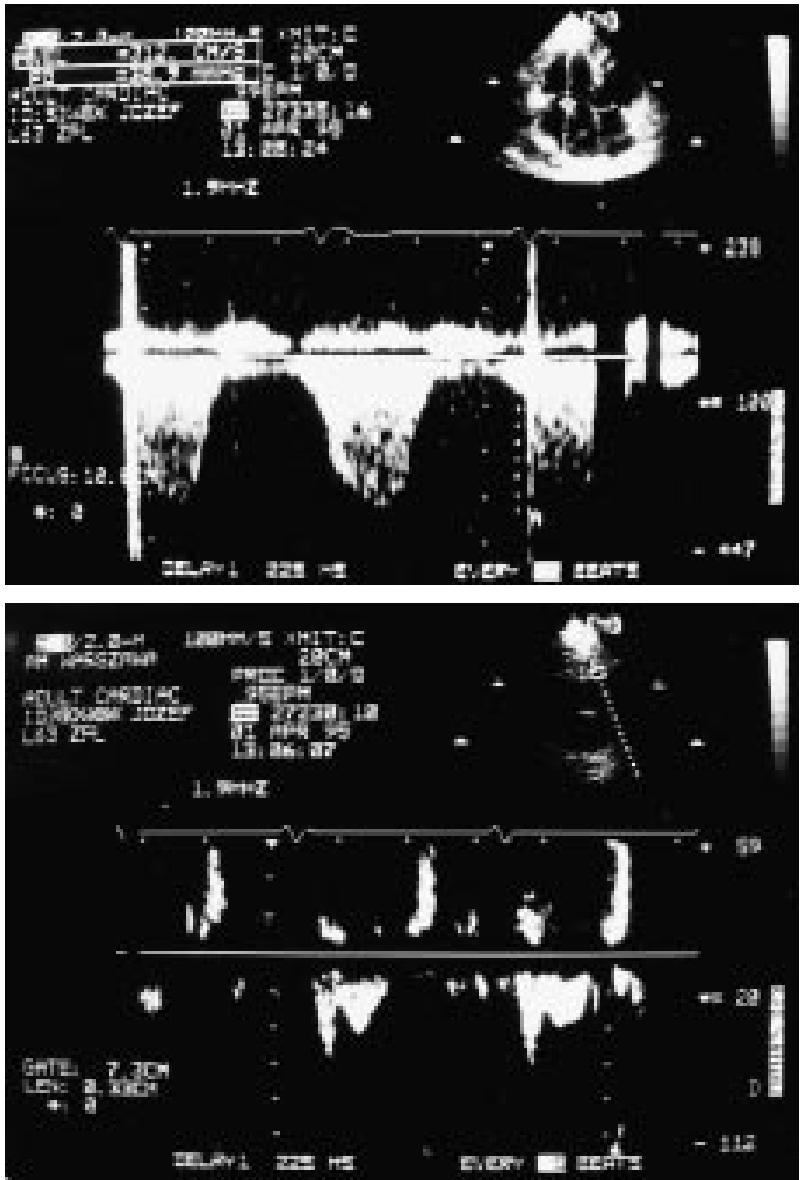

Fig. 3. - An example of "acceleration time $(\operatorname{tacc})<60$, tricuspid valve pressure gradient (TVPG) $<60 "$ sign in a 63 -yr-old patient with acute proximal pulmonary embolism (AP-PE). Despite only moderate elevation of TVPG (39 $\mathrm{mmHg})$, the systolic flow-velocity curve (SVFC) displays prominent and early midsystolic deceleration with very short tacc $(37 \mathrm{~ms})$.

wall filter settings and reproducibility of tacc measurements may vary between centres. Prospective studies are needed to confirm these findings as well as to provide data on SFVC in other clinical situations such as acute respiratory distress syndrome (ARDS), although, in the authors' experience, patients with ARDS do not seem to cause SFVC abnormalities comparable to those in AP-PE.

\section{Limitations of the study}

Direct haemodynamic measurements were not performed in all patients. This applies especially to AP-PE. However, this does not affect the main clinically relevant finding of this study, namely the dissociation of results derived from the two most commonly used noninvasive tests for assessment of $P$ pa. It is possible that AP-PE may have caused acute RV failure and resulted in a rise of right atrial pressure with less of an increase in TVPG than would have been expected. Similar diameters of IVC, RVedd and similar mean cardiac frequencies in the four studied groups seem to indicate that the differences in the degree of RV failure were not very important. Furthermore, even if underestimated by TVPG, the systolic $P$ pa in acute pulmonary embolism should not exceed $60 \mathrm{mmHg}$, limited by 
the maximum output of a nonhypertrophied RV. This would still be well below pressures found in CP-PE and PPH in this study. Also, the validity of TVPG assessment of systolic $P$ pa in a wide spectrum of clinical settings, including acute pulmonary embolism, has been well documented previously $[1-4,10]$.

It could be thought that the striking abnormalities of SFVC in patients with AP-PE were due to acute RV failure rather than to the interference caused by the reflected pressure wave. This cannot be directly answered by this study, although the similarity of SFVC in the two groups with proximal thromboemboli, regardless of the severity of pulmonary hypertension and its mode of onset, seems to contradict such a hypothesis. More importantly, early midsystolic deceleration was observed by FURUNO et al. [12] only in dogs with proximal pulmonary arterial constriction and not distal embolization, although in both subgroups pulmonary hypertension was induced acutely, reached similar levels and resulted in acute RV failure. In a recent study by NAKAYAMA et al. [22], important differences in the pulmonary pressure waveform between patients with $\mathrm{PPH}$ and those with pulmonary hypertension due to proximal chronic thromboembolism were found. The large amplitude of pulse pressure in patients with severe chronic thromboembolic pulmonary hypertension was attributed to the thrombi attached to the proximal arteries, narrowing their lumen size and possibly stiffening the arterial wall. A similar phenomenon has also been reported in acute pulmonary embolism despite lower levels of pulmonary hypertension, and has been referred to as "ventricularization" of the pulmonary pressure curve [23]. This confirms the similarities in the pattern of coupling of RV to pulmonary circulation in the presence of proximal emboli irrespective of the level and mode of onset of pulmonary hypertension.

A wall filter is necessary when recording SFVC to decrease the background noise and allow for subsequent measurements. This procedure removes a small initial part of the flow-velocity curve close to the baseline and thus artificially decreases tacc. However, as extrapolation was avoided (fig. 1) and the filter kept low, these results should not be biased in this respect. In fact, the applied method of measurement would tend to underestimate the existing differences in tacc between patients with and without proximal pulmonary embolism.

Why have the striking abnormalities found in AP-PE not been previously reported considering the $15 \mathrm{yrs}$ of assessing tacc in clinical practice? Strange as it seems, there were virtually no published reports dealing with SFVC in patients with pulmonary embolism. It is suspected that in many cases, tracings displaying profound and early midsystolic deceleration, together with relatively low flow velocity due to poor RV output and distended RVOT might have been mistaken for artefacts and considered as unsuitable for analysis (fig. 2). This was, in fact, the authors' initial attitude. However, these "poor quality" tracings were found much too often despite good quality two-dimensional echocardiographic images of the RVOT and pulmonary valve. The "artefacts" were also highly reproducible. Knowing the type of the pattern that might be expected and using appropriately low wall filter and scale settings allows SFVC to be recorded in most patients with AP-PE.

\section{Conclusions}

Important divergence in the results of two established noninvasive Doppler methods of estimation of $P$ pa based on tricuspid jet velocity and flow-velocity curve in the RVOT, respectively, was found between patients with various causes of cor pulmonale. Despite a relatively mild increase in systolic $P$ pa as indicated by the TVPG, the most disturbed SFVCs were found in patients with AP-PE, and were characterized by early midsystolic deceleration and shortest $t$ acc. In general, in a studied group of patients with AP-PE, CP-PE, CPLD, and PPH, the presence or absence of proximal thromboemboli and not the level of pulmonary hypertension seemed to be the major determinant of SFVC. This is believed to be caused by the early return of the reflected pressure wave interfering with RV ejection. Whenever short tacc $(<60 \mathrm{~ms})$ is found in the presence of no more than a moderate increase of TVPG $(<60 \mathrm{mmHg})$ as assessed by the echo/Doppler, AP-PE should be strongly considered, although assessment of the specificity and sensitivity of this sign requires prospective studies.

It is suggested that acceleration time duration and the presence of midsystolic deceleration carry important information regarding the dynamic coupling of the right ventricle to the pulmonary arterial bed. While potentially useful for evaluation of true right ventricular afterload during pulsatile flow conditions, the systolic flow velocity curve may not provide a reliable estimate of pulmonary arterial pressure.

\section{References}

1. Currie PJ, Seward JB, Chan KL, et al. Continuous wave Doppler determination of right ventricular pressure: a simultaneous Doppler-catheterization study in 127 patients. J Am Coll Cardiol 1985; 6: 750-756.

2. Chan KL, Currie PJ, Seward JB, Hagler DJ, Mair DD, Tajik AJ. Comparison of three ultrasound methods in the prediction of pulmonary artery pressure. $\mathrm{J} \mathrm{Am}$ Coll Cardiol 1987; 9: 549-554.

3. Laaban JP, Diebold B, Zielinski R, Lafay M, Raffoul H, Rochemaure J. Noninvasive estimation of systolic pulmonary artery pressure using Doppler echocardiography in patients with chronic obstructive pulmonary disease. Chest 1989; 96: 1258-1262.

4. Chapoutot L, Metz D, Jolly D, et al. Diagnostic prognostic and therapeutic value of Doppler echocardiography in pulmonary embolism. A propos of 41 cases. Ann Cardiol Angiol 1989; 38: 523-529.

5. Kitabatake $\mathrm{A}$, Inoue $\mathrm{M}$, Asao $\mathrm{M}$, et al. Non-invasive evaluation of pulmonary hypertension by a pulsed wave Doppler technique. Circulation 1983; 68: 302-309.

6. Kosturakis D, Goldberg SJ, Allen HD, Loeber C. Doppler echocardiographic prediction of pulmonary arterial hypertension in congenital heart disease. Am J Cardiol 1984; 53: $1110-1115$.

7. Isobe M, Yazaki Y, Takaku F, et al. Prediction of pulmonary arterial pressure in adults by pulsed Doppler echocardiography. Am J Cardiol 1986; 57: 316-321.

8. Marchandise B, De Bruyne B, Delaunois L, Kremer R. Noninvasive prediction of pulmonary hypertension in chronic obstructive pulmonary disease by Doppler echocardiography. Chest 1987; 91: 361-365.

9. Marangoni S, Quadri A, Dotti A, et al. Non-invasive assessment of pulmonary hypertension: a simultaneous echo-Doppler study. Cardiology 1988; 75: 401-408. 
10. Torbicki A, Skwarski K, Hawrylkiewicz I, Pasierski T, Miskiewicz Z, Zielinski J. Attempts at measuring pulmonary arterial pressure by means of Doppler echocardiography in patients with chronic lung disease. Eur Respir $J$ 1989; 2: 856-860.

11. Torbicki A, Tramarin R, Morpurgo M. Role of echo/ Doppler in the diagnosis of pulmonary embolism. Clin Cardiol 1992; 15: 805-810.

12. Furuno Y, Nagamoto Y, Fujita M, Kaku T, Sakurai S, Kuroiea A. Reflection as a cause of mid-systolic deceleration of pulmonary flow wave in dogs with acute pulmonary hypertension: comparison of pulmonary artery constriction with embolization. Cardiovasc Res 1991; 25: 118-124.

13. Pruszczyk P, Torbicki A, Chlebus $\mathrm{M}$, et al. Noninvasive diagnosis of suspected severe pulmonary embolism: transesophageal echocardiography vs. spiral CT. Chest 1997; 112: 722-728.

14. Torbicki A, Tramarin R, Fracchia C, Mortara A, Ambrosino N, Pozzoli M. Effect of increased right ventricular preload on pulmonary artery flow velocity pattern in patients with normal or increased pulmonary artery pressure. Am J Noninvas Cardiol 1994; 8: 151-155.

15. Tramarin R, Torbicki A, Marchandise B, Laaban JP, Morpurgo M. European cooperative study on echo-Doppler evaluation of pulmonary artery pressure in patients with chronic lung disease. Eur Heart $J$ 1991; 12: 103-111.

16. Torbicki A, Tramarin R, Fracchia C, et al. Reliability of pulsed wave Doppler monitoring of acute changes in pulmonary artery pressure in patients with chronic obstructive pulmonary disease. In: Widimsky J, Herget J, eds. Pulmonary Blood Vessels in Lung Disease. Basel, Karger, 1990; pp. 133-141.

17. Torbicki A. Natural variability of echo-doppler parameters. In: Doppler Echocardiography in Chronic Lung Diseases. PhD thesis. Warsaw, Warsaw University School of Medicine, 1991; pp. 96-99.

18. Piene H. Pulmonary arterial impedance and right ventricular function. Physiol Rev 1986; 3: 606-652.

19. Kussmaul WG, Noordegraaf A, Laskey WK. Right ventricular-pulmonary arterial interactions. Ann Biomed Eng 1992; 20: 63-80.

20. Laskey WK, Ferrari VA, Palevsky HI, Kussmaul WG. Pulmonary artery hemodynamics in primary pulmonary hypertension. J Am Coll Cardiol 1993; 21: 406-412.

21. Turkevich D, Groves BM, Micco A, Trapp JA. Early partial systolic closure of the pulmonic valve relates to severity of pulmonary hypertension. Am Heart J 1988; 115: 409-418.

22. Nakayama $\mathrm{Y}$, Nakanishi $\mathrm{N}$, Sugimachi $\mathrm{M}$, et al. Characteristics of pulmonary artery pressure waveform for differential diagnosis of chronic pulmonary thromboembolism and primary pulmonary hypertension. $\mathrm{J} \mathrm{Am}$ Coll Cardiol 1997; 29: 1311-1316.

23. Morpurgo M, Merzagalli M, Casazza F. "Ventricularization" of the pulmonary artery pressure curve: a hemodynamic sign of proximal pulmonary embolism. Cardiologia 1998; 43: 745-748. 